\title{
Keine okkulten Hirnschäden durch Migräne-Aura
}

\author{
In einer großen Zwillingsstudie aus Dänemark ergab sich kein erhöhtes Risiko für klinisch stumme \\ Hirninfarkte oder White Matter Lesions bei Patienten, die unter Migräne mit Aura leiden.
}

\begin{abstract}
_ Dass Migräne mit Aura mit einem erhöhten kardiovaskulären Risiko assoziiert ist, ist gut dokumentiert. Aufsehen erregte die niederländische CAMERAStudie, in der sich auch ein erhöhtes Risiko für klinisch stumme Hirninfarkte und vermehrte White Matter Lesions (WML) zeigte. [JAMA. 2004;291:42734; Cephalalgia. 2010;30:129-36; JAMA. 2012;308:1889-97]. Diese Ergebnisse konnten bisher nicht repliziert werden. Eine Arbeitsgruppe aus Dänemark versuchte es nun nochmals.

Dafür wurden Frauen aus dem dänischen Zwillingsregister rekrutiert. Von 1.694 Personen, die auf den initialen Fragenbogen antworteten, wurden 172 Migränepatientinnen mit Aura einge-
\end{abstract}

schlossen. Außerdem nahmen 34 zugehörige Zwillinge ohne Migräne teil. Als weitere Kontrollpersonen wurden 139 Frauen aus dem Register, die gar nicht von Migräne betroffen waren, rekrutiert. Bei allen wurden 3-Tesla-Magnetresonanztomografien durchgeführt und verblindet ausgewertet. Die Frauen waren im Mittel 48 Jahre alt. 40\% waren monozygote Zwillinge. Vaskuläre Risikofaktoren waren gleichmäßig verteilt.

Stumme Hirninfarkte fanden sich bei vier Migränepatientinnen und einer Kontrollperson. Dieser Unterschied war nicht signifikant. Auch bei der Ausprägung und der Häufigkeit von WML ergaben sich unter Verwendung verschiedener Scores keine Unterschiede.
- Gaist D, Garde E, Blaabjerg M et al. Migraine with aura and risk of silent brain infarcts and white matter hyperintensities: an MRI study. Brain. 2016;139:2015-23

\section{KOMMENTAR}

Die Ergebnisse dieser großen populationsbezogenen Studie an weiblichen Zwillingen weichen sowohl in Bezug auf die stummen Insulte als auch auf die WML von $\operatorname{der}$ CAMERA-Studie ab. Die dänische Studie ist größer als die niederländische - und gerade in der Subanalyse der teilnehmenden Zwillingspaare wird eine unterschiedliche Prävalenz von vaskulären Risikofaktoren verhindert. Der einzige Nachteil der dänischen Studie ist, dass es sich um eine Querschnitts- und nicht um eine Längsschnittstudie handelt.

Prof. Dr. med. H.-C. Diener

\section{Parallele intra- und extrauterine Schwangerschaft}

Eine 34-jährige Frau mit Zustand nach Appendektomie vor vielen Jahren stellte sich mit starken Unterbauchschmerzen seit ca. einer Stunde in der Nothilfe vor. Sonografisch war einige Tage zuvor eine orthotop gelegene Schwangerschaft in der achten Gestationswoche festgestellt worden. Bei der Untersuchung war die Patientin hypoton und tachykard und wies einen deutlichen Druckschmerz im rechten Unterbauch auf. Die transvaginale Sonografie zeigte eine $4 \times 4 \mathrm{~cm}$ große Raumforderung im Bereich der rechten Adnexe (Abb. A) sowie freie Flüssigkeit in der Bauchhöhle (Abb. B). Dieser Befund weckte den Verdacht auf eine ektope Schwangerschaft, sodass notfallmäßig eine Laparoskopie durchgeführt wurde. Dabei erkannte man tatsächlich eine ektope Schwangerschaft, die zu einer Ruptur des rechten Eileiters ge- führt hatte (Abb. C). Der Dottersack war intakt und auch der Embryo erkennbar. Eine aktive Blutung im Bereich des rupturierten Eileiters konnte durch eine Salpingektomie gestoppt werden.

Eine gleichzeitig vorliegende intra- und extrauterine, also heterotope Schwangerschaft ist sehr selten. Die Inzidenz bei natürlicher Empfängnis wird auf $1: 30.000$ Schwangerschaften geschätzt. Im vorliegenden Fall blieb die intrauterine Schwangerschaft intakt, und die Patientin gebar termingerecht ein gesundes Mädchen.

Prof. Dr. med. H. S. FüeßI

- Goettler S, Zanetti-Dällenbach R (rosanna.zanetti@unibas.ch). Heterotopic pregnancy. NEngl J Med. 2016;375:1982
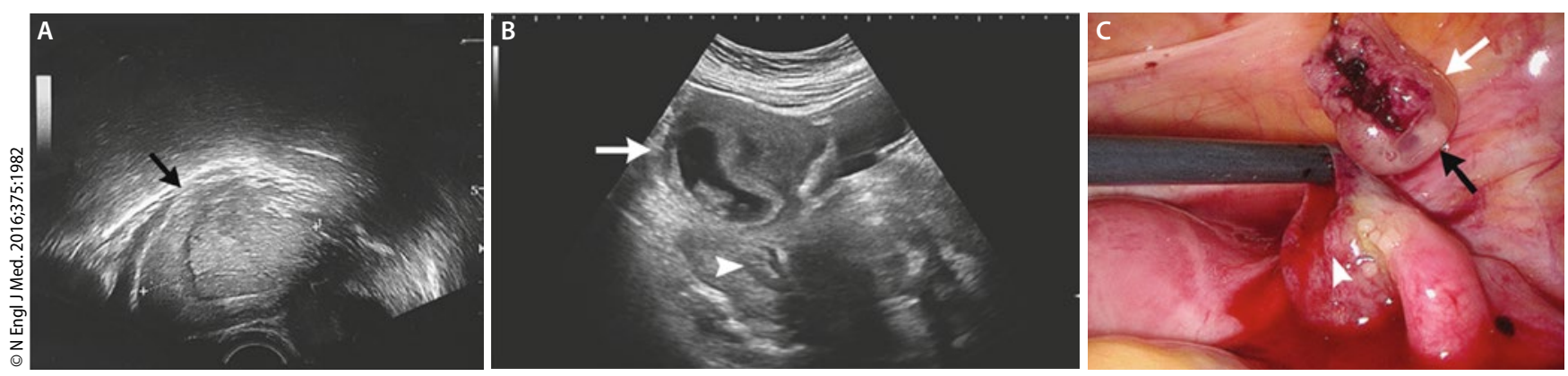

A: Raumforderung im Bereich der Adnexe. B: Intrauterine Schwangerschaft (Pfeil) und freie Flüssigkeit (Pfeilspitze).

C: Rupturierter Eileiter (Pfeilspitze), extrauteriner Dottersack (weißer Pfeil), Embryo (schwarzer Pfeil). 